\title{
INTERNATIONAL COMPARISONS
}

\section{Health care of American children}

\author{
Ross Mitchell
}

The use of infant mortality rates, perinatal mortality rates, and other population measures as the basis for comparison of child health trends in different countries is familiar to us all. Differences in rate are usually attributed largely to variations in nutrition, housing, and public health policies. Though socioeconomic factors are undoubtedly important as determinants of child health, the provision, efficacy, and uptake of health services cannot be dismissed as of little significance. Perhaps national differences in the delivery of health care have not attracted much attention because most countries are preoccupied with their internal arrangements and tend to believe that other systems are unsuitable or not applicable to their own situation. Concern about the deteriorating position of the USA vis-à-vis some other developed countries in such areas as immunisation against contagious diseases, care of acute illness as reflected in postneonatal infant mortality rates, unintentional injury, and care of children with special health needs, however, stimulated the American Academy of Pediatrics (AAP) to probe deeply into the reasons for the relatively poor American performance and to invite comparisons from other developed countries (Canada, England and Wales, France, Norway, and the Netherlands). The resultant conference and its published report documented the necessity for the USA to address the needs of all its children, to establish priorities, and to set goals. ${ }^{1}$

In all four of the areas of child health examined in the AAP report, America compared unfavourably with the other countries. Thus deaths from accidents and postneonatal infant mortality rates were both worse than in Europe and services for children with special health needs were not so widely accessible. The incidence of contagious diseases, still a major cause of death in developing countries, declined precipitously in the USA during the past half century because of a successful immunisation policy. However, rates of routine immunisation of preschool children are now falling and unvaccinated children are increasingly at risk. There is no readily identifiable reason for this, though inadequate health insurance and the rising cost of vaccines are thought to play a part.

Immunisation rates of 55 to $65 \%$ for selected diseases now compare with rates of 80 to $100 \%$ in certain northern European countries and 76 to $87 \%$ in England and Wales. The rates in the USA vary widely between ethnic groups but even the highest rates among white Americans are well below those of the European countries. In England and Wales, despite a relatively favourable situation, national immunisation rates of over $90 \%$ have not yet been achieved and there is considerable regional and social variation, so sustained effort and continuing vigilance are essential both in the UK and in the USA.

With all the ideas advanced to explain these and other national differences, it is important to bear in mind the diversity of provision within the USA. All the other countries considered have more uniformity in the organisation of services because they have greater unity of political structure, with a substantial degree of central control of policies and performance. In the USA, where sizable autonomy is exercised by each state, there is a much wider range of service quality, from the excellent to the poor, and national figures reflect this variety. Statistics do not tell the whole story, for the greater the degree of control the larger the potentiality for better figures but the less the opportunity for personal choice. Too ardent a devotion to improving statistics may therefore sometimes result in unacceptable restriction of individual freedom, as sometimes appears to be a risk in the maternity services. Nevertheless, there seems no doubt that the relatively poor figures in the USA are the result of fragmentation and duplication of services with too many gaps, of economic disincentives, and of failure to act decisively in such matters as traffic regulations and firearms control.

Some of the agonising of the Americans may be misplaced. For example, the President of the AAP, Birt Harvey, deplores the tendency in the USA to look at children as chattels of their families, while other developed countries consider their children to be a national resource. Would that this were true of the UK, which is far from being a childcaring society! Harvey is disproportionately hard on the record in the USA when he compares child abusing Americans with the European peoples who 'tend to treasure and care for all children'. Child abuse statistics from Europe do not bear out this idyllic view and indeed Henry Kempe always said that child abuse would be found in every country if only people looked for it.

Comparison of the USA with a group of other developed countries tends to lump the latter together, obscuring differences between them and creating an artificial 'us and them' antithesis. Thus, for example, the laxity of laws on drunk driving is said to illustrate lower standards in the USA, yet these laws, extremely strict in Scandinavia for many years, have only recently been tightened up in the UK, which still lags behind other European countries.

Canada might be thought to afford the closest comparison with the USA, because they share a North American history and culture. The 
Canadian health care system is a compromise between 'fee for service' and socalled 'socialised medicine' and the balance is claimed to be remarkably successful. In Canada, though responsibility for health is shared between federal and provincial governments, the Federal Department of National Health and Welfare serves, in the words of Ivan Pless, as 'the spiritual guardian of the integrity of Canada's health services'. The principle of a two class system of health care, one for the rich and another for the poor, does not exist anywhere in Canada. Whereas in the UK families are means tested to establish their entitlement to milk and vitamins, help with travelling costs to hospital and other benefits, in Canada no demeaning test determines the availability of health care. In that country, they really believe that equitable access to medical care is essential to the integrity of a fair and just society, whereas the USA fails to guarantee adequate care for all children because eligibility and benefits are so variable. ${ }^{2}$

England and Wales, along with the rest of the UK, have had a National Health Service for over 40 years, originally based on the principle of free and equal access to health care for all but presently in process of change towards a compromise system between a state service and free enterprise. ${ }^{3}$ The effects on child health of the changes being introduced are imponderable but among fears expressed are that children with chronic disease or disability will be at a disadvantage because the cost of their continuing care will weigh too heavily on the budgets of family doctors. Moreover, there is incongruity between a system of buying and selling hóspital services on a contractual basis and the concept of a single planned hospital service for children, whose needs are so different from those of adults, and there is enormous potential for divisive duplication. ${ }^{4}$ On a more optimistic note, the adverse effects of mercenary policies and persistent underfunding may be offset by effective integration of the child health services in each region and district, linking together preventive services, acute services, and services for the chronically disabled or ill child. Such coordination of health programmes to meet children's needs was advocated by the Court committee $^{5}$ but has not yet been fully implemented. ${ }^{6}$ Attempts to improve resource management and medical audit are laudable but might more sensibly have been undertaken in the context of a free and universally available service. In a system of bargaining for services from various separate providers, ${ }^{7}$ a danger is that administration, already time consuming, becomes an end in itself and much more expensive than the cost of administering the present health service. In such circumstances, despite government protestations that the patient comes first, the real needs of children tend to be lost sight of amid the havoc that competition brings to health care. ${ }^{8}$ There is thus a tension between conflicting philosophies in the health service in the UK and only time will tell whether or not the new patterns of child health care will be to the greater benefit of children and their families.

Every system of child health care has its strengths and weaknesses. For example, the strengths of the French system are the universal health record (carnet de santé), recording specific information at selected ages, and the specialised Centres for Early Medico-Social Action. Weaknesses are the shortage of staff and resources in the school health service and unevenness in availability of some social supports. Similar strengths and weaknesses could be pointed out for all the national health systems considered in the AAP report. The willingness of Americans to expose their deficiencies and to indulge in honest and frank soul searching is admirable, for many countries in the world have neither the courage to do so nor the hard data from which to draw conclusions.

The balance between individual freedom and community aims is complex and will vary between countries and between states in a federal system, depending on such variables as historical development, political philosophies, national temperament, and cultural patterns. Community aims lay great store on protecting the wellbeing and security of its weaker members and this necessarily means curbing individual rights in some degree. For example, in most countries, though apparently not in America, the safeguarding of a woman's employment during and after pregnancy involves imposing conditions on employers. Children are vulnerable and cannot speak for themselves, so that the scales should be tilted from personal liberty towards community responsibility when child health services are being considered. A fundamental characteristic of the American health system is the freedom of the individual to choose, wisely or unwisely. However, the Canadian experience provides cogent reasons why the USA should move towards a more planned and regulated system of universal health care and this recommendation is made in the AAP report, which observes that a decade of trying market systems based on individual responsibility shows that these approaches have not worked. It is ironic, therefore, that at the same time the UK is moving towards a competitive market system, especially as the Canadian example suggests that deregulation and competition tend to increase costs and diminish the quality of health care. Moreover, the burgeoning of private medicine is unlikely to improve child health, for in the French system, which combines public and private components, there is a notable weakness of information for statistical analysis coming from the private sector-a salutory observation for those advocating a move towards private care.

The difficulties of providing for minority populations, especially underprivileged and migrant groups, are evident in all countries and are especially emphasised by Manciaux. ${ }^{9}$ Disparity in health between socioeconomic groups within a country is often of greater importance than small differences between total population rates for comparable nations. Thus there is evidence that in France the differential in infant mortality between the social extremes, while still too wide, is narrowing. By contrast, in New York City the neonatal and infant mortality rates in disadvantaged urban com- 
munities have risen dramatically in the past few years. ${ }^{10}$ In the UK, where some four million children live in poverty, the health gap between the affluent and the poor is known to be widening. Clearly both the USA and the UK should study the variations in rates between different sections of their own child populations if the aim is to improve overall statistics. Such scrutiny will demand action and action costs money: no extra money means no resources for these children or their families.

Relative national costs of health care were not closely examined in the AAP report but it was observed that the per capita health bill in the USA (over $11 \%$ of gross national product) may be too high in relation to the services provided and the results achieved. It is $41 \%$ higher than Canada's expenditure and $85 \%$ more than France's, but the fact that it is $171 \%$ more than Britain's should give us food for thought about our priorities in the allocation of national resources. Even if total financial provision for the health service were to be increased, there is no certainty that children would receive the priority that such a precious national asset should demand. As Sverre Lie says, speaking of the Norwegian health services, 'for children there will be a constant fight to retain a reasonable share of the cake' and this is true for all countries and all systems.

The AAP report concludes that the USA must change its philosophy about social welfare and about looking after children. Steps are already being taken towards improving the health and welfare of all children but it is conceded that the end of this road will not be reached for many years. Nevertheless, despite all the self criticism and heart searching, the reader cannot but reflect how fortunate is a child living in the USA, or indeed in any of the countries considered, in comparison with a child born in one of many countries in Asia, Africa, or South America, whose heritage is destitution and famine, whose quality of life is often grim, and whose fate is likely to be malnutrition, disease, and all too often early death.

1 American Academy of Pediatrics. Child health in 1990: the United States compared to Canada, England and Wales, France, the Netherlands and Norway. Pediatrics 1990;86: suppl 1025-127.

2 Harvey B. Why we need a national child health policy Pediatrics 1991;87:1-6.

3 Chantler C. Paediatricians and the new National Health Service. Arch Dis Child 1991;66:357-60.

4 Appleyard WJ. Children's services in the new NHS-a struggle for survival. Arch Dis Child 1990;65:635-7.

5 Court SDM, chairman. Fit for the future. The Report of the Court SDM, chairman. Fit for the future. The Report of the
Committee on Child Health Services. London: HMSO, Committee on Child
1976. (Cmnd 6680.)

6 Macfariane A, Mitchell R. Health services for children and their relationship to the educational and social services. their relationship to the educational and social services. In: Forfar JO, ed. Child health in a changing
Oxford University Press, 1988: 155-97.

7 Plamping D. The new NHS. BMF 1991;302:737-8.

8 Light DW. Perestroika for Britain's NHS. Lancet 1991;337. 778-9.

9 Manciaux M. Children of disadvantaged families. Contemporary studies in Western Europe. Paris: UNESCO, 1981 (SS 81/WS/48.)

10 Heagarty MC. America's lost children: whose responsibility? J Pediatr 1991;118:8-10.

\section{Campylobacter jejuni and Guillain-Barré syndrome}

Although it has long been known as postinfective polyneuritis it is unusual to demonstrate a specific infectious aetiology in GuillainBarré syndrome. Recently, however, there have been several reports linking the syndrome with Campylobacter jejuni. A paper from Japan (Shigekazu Kuroki and colleagues, The Pediatric Infectious Disease Fournal 1991;10:149-51) reports on 11 patients aged 7-14 years with Guillain-Barré syndrome seen over a five year period. Stool culture was performed in eight of them and $C$ jejuni isolated in seven of whom five had had diarrhoea or abdominal pain or both. Serotyping was done in four cases and all four had $C$ jejuni type 19 a serotype previously found in only $1 \cdot 7 \%$ of cases of $C$ jejuni enteritis in Japan. In a previous survey $C$ jejuni was isolated from the stools in $1 \cdot 7 \%$ of healthy children. The authors found no evidence to support a previous suggestion that Guillain-Barré syndrome might be more severe when associated with $C$ jejuni infection.

A stool culture should be included in the diagnostic work up of children with Guillain-Barré syndrome. 